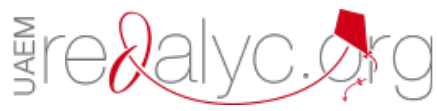

Centro Sur

ISSN: $2600-5743$

compasacademico@icloud.com

Grupo Compás

Ecuador

\title{
La desidia por la educación media-superior
}

Gordillo-Parrales., Pedro Luis

La desidia por la educación media-superior

Centro Sur, vol. 2, núm. 2, 2018

Grupo Compás, Ecuador

Disponible en: http://www.redalyc.org/articulo.oa?id=588861693004

Esta obra está bajo una Licencia Creative Commons Atribución-NoComercial-SinDerivar 4.0 Internacional. 


\title{
La desidia por la educación media- superior
}

\author{
Laziness for upper-middle education \\ Pedro Luis Gordillo-Parrales. pgordillo@itsvr.edu.ec \\ Instituto Superor Vicente Rocafuerte, Ecuador \\ http://orcid.org/0000-0002-3281-0185
}

Centro Sur, vol. 2, núm. 2, 2018

Grupo Compás, Ecuador

Recepción: 18 Enero 2017

Aprobación: 26 Julio 2018

Redalyc: http://www.redalyc.org/ articulo.oa?id $=588861693004$

\section{BY-NC-ND}

Resumen: El presente trabajo de investigación surgió de una inminente preocupación, cuya finalidad era investigar por qué, los jóvenes presentan gran apatía o rechazo hacia la educación y razón por la que investigadores manifiestan, que la educación está en terapia intensiva, que no solo debe preocupar a una nación, sino a las autoridades educativas, docentes, familias y alumnos, ya que su efecto está generando consecuencias nefastas en el campo educativo y en el futuro del conglomerado juvenil . Basado en esta inquietud se inició la indagación con la necesidad de identificar los factores que determinen el poco interés de los educandos hacia los estudios. Para realizar la investigación se planteó como objetivo, determinar los factores que ocasionan la desidia, por la educación media y superior en jóvenes y adolescentes. Para ello, se realizó un estudio analítico y reflexivo de 50 artículos relacionados con el tema, considerado problema mundial que afecta a los estudiantes incrementándose de forma incontrolable. El diseño de esta investigación es de tipo descriptivo y bibliográfico. Los resultados señalan como responsables a los factores socio-económicos-culturales y en determinados casos sociopersonales. En conclusión, se debe jerarquizar la educación, para que sus efectos positivos permitan llegar al umbral del conocimiento, con la finalidad de crear valores, derechos educativos, futuro laboral y bienestar socio-económico colectivo.

Palabras clave: Desidia , Jóvenes adolescentes, calidad educativa, educación familiar.

Abstract: The present research work emerged from an imminent concern, whose purpose was to investigate why, young people show great apathy or rejection of education and why researchers say that education is in intensive care, which should not only concern a nation, but to the educational authorities, teachers, families and students, since its effect is generating disastrous consequences in the educational field and in the future of the youth conglomerate. Based on this concern, the investigation began with the need to identify the factors that determine the little interest of the students towards the studies. In order to carry out the research, the objective was to determine the factors that cause neglect, for secondary and higher education in young people and adolescents. To do this, an analytical and reflective study of 50 articles related to the subject was carried out, considered a global problem that affects students increasing uncontrollably. The design of this research is descriptive and bibliographic. The results indicate the responsible socio-economic-cultural factors and in certain socio-personal cases. In conclusion, education must be hierarchized, so that its positive effects allow reaching the threshold of knowledge, with the purpose of creating values, educational rights, future work and collective socio-economic welfare.

Keywords: Desidia, Young Adolescents, Educational Quality, Family, Education.

\section{INTRODUCCIÓN}

Mediante una reflexión teórica el presente artículo, pretende resaltar algunos aspectos muy significativos referente al desinterés por la educación en la población juvenil, la cual se ha convertido en un fenómeno que está afectando en la mayoría de las instituciones educativas 
de todas las naciones del mundo. Por tal razón, en la actualidad se considera, que la educación está en terapia intensiva, requiriendo atención urgente y especializada, motivo para que nos incite a la reflexión por sus consecuencias futuras, por eso resulta prioritario asumir el estudio de esta investigación, porque sus efectos ya están lesionando gravemente al colectivo de jóvenes sumiéndolos en la desesperanza.

La importancia de este trabajo Investigativo, tiene como objetivo principal, determinar los principales factores que ocasionan la desidia en jóvenes y adolescentes por la educación media y superior. Para el estudio se revisaron artículos relacionados con la problemática, siendo necesario, la selección, análisis y síntesis del contexto, que nos permita entender causas efectos y resultados donde algunos autores resaltan la importancia del tema. La situación resulta compleja, porque las conclusiones determinan que las causas son múltiples, entre las más relevantes se atribuyen a la educación familiar y la calidad educativa, que están ocasionando grave deterioro Psíco- socio- emocional, en la juventud.

Por esta razón, se hace énfasis, al desinterés que tienen determinados jóvenes hacia la educación, representando un serio dilema, no solo para el Estado, sino también para las Instituciones Educativas, Docentes, Padres de Familia y Alumnos en general, es decir, involucra a toda la sociedad en conjunto. En realidad, es preocupante, porque la educación, es la clave para enfrentar las profundas inequidades que pueden ocurrir en el proceso de la vida, por eso es considerada la puerta hacia el exterior, que permitirá la superación de los individuos, constituyéndose como la base fundamental en el desarrollo tecnológico, científico e innovador para los avances de una nación.

Cabe recalcar, que para enfrentar ese mundo exterior, deben estar preparados siendo imprescindible el compromiso y trabajo constante, por parte de los padres de familia y educadores, cuya finalidad debe estar orientada hacia la educación, reiterándoles que la enseñanza aprendizaje constituye el pilar básico que conduce hacia el éxito . Desde este punto de vista se debe insistir que los actores responsables, no deben desmayar en sus propósitos para evitar que la juventud caigan en errores, tropiecen con obstáculos que puedan ocasionarle daño y se auto destruyan, además señalarles cómo superar las dificultades para poder disfrutar de la vida.

La Organización de las Naciones Unidas (ONU) en 1989, aprobó la aplicación de los derechos de los niños como inalienables, el derecho a la educación, protección, alimentación, seguridad y no a la discriminación.

La Organización de Naciones Unidas para la Educación, la Ciencia y la Cultura (UNESCO) en el 2012, advierte y pone en alerta sobre el inminente peligro de la deserción escolar, y señala que existen 6,5 millones de niños en América Latina que no asisten al colegio para ejercer su derecho a la educación. En la misma línea el organismo internacional, aseguró que 15,6 millones de infantes asisten a los centros educacionales, en medio de un clima y entorno de vulnerabilidad que pone en riesgo su continuidad en el proceso educativo. Además señala que la educación es un derecho, con respeto, justicia social, sin discriminación, pero a pesar de todo lo antedicho, en ciertos países lo asimilan a su manera. 
En América latina y el Caribe, la UNESCO, según últimos estudios revela que cerca de 263 millones de niños, adolescentes y jóvenes de todo el mundo no están escolarizado, es decir, que vivimos una crisis de aprendizaje, porque uno de cada seis alumnos, no alcanzan niveles mínimos de competencia.

En el Ecuador, el Instituto Nacional de Estadísticas y Censo (INEC) 2010, por intermedio de la Comisión de Educación revela que de cada 100 estudiantes matriculados, 20 abandonan sus estudios desde el octavo de básica sin culminar el bachillerato.

Por todo lo expuesto y de forma general tenemos que la desidia por la educación, está ocasionando efectos negativos y sus consecuencias serán dramáticas, porque uno de los resultados más alarmantes es la falta de competitividad, es decir que no están preparados para enfrentarse al mundo laboral, generando crisis socio económica, que afecta a todas las personas, de forma directa a los jóvenes, porque las oportunidades de empleo estarán en relación al grado de educación y cultura que tenga el postulante, la cual sino está capacitado, ni actualizado, no estará apto para el desempeño de la actividad propuesta, con el consecuente incremento del subempleo y desempleo. En conclusión tenemos, que aquellos que no terminaron sus estudios medios y superiores, tendrán las peores ofertas laborales y mala remuneración, en cambio los que lograron culminar sus estudios, tendrán mejores oportunidades de trabajo, bien remunerado ya sean estables o simplemente contratados.

\section{MATERIALES Y MÉTODOS}

Para realizar el proceso del trabajo investigativo, ha sido necesario aplicar técnicas, métodos y procedimientos pertinentes, con la finalidad de elaborar, definir, sistematizar y evaluar las acciones aplicadas con la posible solución a una problemática

El presente trabajo de investigación se enmarca en la modalidad de tipo cualitativa, que nos permitió comprender y describir las características del objeto y campo de estudio, porque a través de ella, se identificó el problema, basado en contextos estructurales, situacionales y su interrelación. Así mismo, es una investigación de tipo exploratoria, necesaria para buscar y centrarse en el problema que se pretende estudiar y conocer, para la producción de conocimientos que nos interesan, considerado un estado incipiente para el sustento de un estudio. Es un tipo de diseño descriptivo, por tratarse de un tema científico, donde se requiere, la visión general del sujeto como parte de la problemática, donde deben describirse actores y escenarios. Además, es una investigación explicativa, porque trata de buscar, explicar y dar respuesta a las causas que han generado el problema.

Para fundamentar este trabajo fue necesario realizar una investigación de tipo bibliográfica, la cual se inició con la búsqueda del estudio, en la página de Google académico, soportado en 50 artículos científicos confiables, seleccionados y relacionados con el tema, fecha y edición de la tesis o revista de publicación de editoras acreditadas a nivel internacional, 
priorizando artículos de los últimos 4 años en un aproximado del $80 \%$ y el $20 \%$ restante referido a los años anteriores.

También ha sido necesario aplicar métodos: Deductivo, porque a partir del trabajo investigativo, se han adquirido conocimientos generales, que formarán conocimientos particulares; de Observación, que nos ha conducido al análisis y síntesis del cual se obtiene la información para el desarrollo del presente estudio. Así mismo es factible, confiable y tiene validez, porque se puede llevar a práctica con perseverancia, los datos son escogidos de fuente fidedigna y será válido cuando la conclusión se derive de las premisas.

\section{RESULTADOS}

Para iniciar este trabajo de Investigación, se realizó la búsqueda y descarga de artículos relacionados con la problemática, cuyo motor de búsqueda fue Google Académico, ya que contiene en su base de datos, temas relevantes de editoras científicas reconocidas por su confiabilidad y alto prestigio internacional tales como: Redalyc, Scielo, Latindex, Compas, entre otras. Que mediante el análisis exhaustivo de los investigadores en cada uno de los casos estudiados, nos han permitido orientar y entender, que los factores causales de la desidia en adolescentes y jóvenes por la educación, se debe a: la falta de motivación, baja de recursos económicos, trabajo prematuro, problemas familiares, personales, el entorno educativo, rol de amigos y la auto actitud del estudiante, que pueden conducirlo a la desmotivación paulatina, luego al absentismo y como resultado el abandono escolar. Es decir, el eje central de la problemática, es la motivación, que dependerá mucho de la forma en que se viva en los contextos familiares y escolares.

Que es motivar?.Desde el punto de vista psicológico existen varias definiciones:

-Estado interno o condición que activa el comportamiento y lo orienta hacia una dirección dada.

-Deseo que energiza y dirige el comportamiento hacia un objeto o meta dada.

-Influencia en las necesidades y deseos que afectan, la intensidad y dirección de los comportamientos.

Así mismo, según Malde Modino, la familia es la unión de personas que comparten un proyecto vital de existencia en común, que se supone duradero en el que se generan fuertes sentimientos de pertenencia a dicho grupo, en el cual existe un compromiso personal entre sus miembros y se establecen intensa relación de intimidad, recepción y dependencia (p.16).

De forma específica la familia es considerada como, la primera escuela donde se aprende a relacionarse mediante la comunicación, necesarias para instruir valores, normas y afecto familiar que nos permita desarrollar en el ámbito socio-educativo

Por tal razón, en el contexto familiar y escolar, no debemos olvidar que el hogar, es el sitio donde se inicia la educación que continúa en la escuela, y gracias a esta motivación adquirida, se pretende lograr una integración 
académica y social ya que durante el proceso de la adolescencia, se recibe a un ser humano irreductible, que busca sentirse parte de un todo, capaces de hacer críticas constantes a los adultos, por sus estructuras y normativas a las que se ve presionado.

Los conflictos familiares son efectos negativos al núcleo de la familia y tendrá sus consecuencias. Por esta razón Correa, Rodríguez, Ceballos, y Álvarez, manifiestan que los conflictos son espacios perdurables de desgaste y trances emocionales que afectan su desenvolvimiento en el proceso socio familiar.

"Al hecho de que el joven adolescente se encuentra en una etapa de transformación, Psicológica, Biológica y Emocional, unimos que también se encuentra en un momento de transición de una etapa educativa a otra, se puede considerar el riesgo que manifieste falta de motivación e implique el fracaso escolar y riesgo de abandono del sistema educativo".

Para evitar el abandono escolar de jóvenes y adolescentes, es importante la integración familia - escuela, para que actúen como agentes educativos, que permitan incorporarlo, reconociendo su capacidad intelectual, volitiva y afectiva, conociendo que hay una base Bio-psico-emocional, muy compleja de entender en el proceso de transición, en la educación del adolescente. Para esto necesita de mucha comprensión, ya que de ello dependerá su triunfo o fracaso, porque a través de la etapa educativa tendrán determinadas dificultades como: el bajo rendimiento en la asignaturas que puede conducirlos a la desmotivación causándoles falta de confianza y baja autoestima, problemas de salud ya sean propios o familiares que puede conducirlos a la inasistencia, dificultad para cumplir tareas y adquirir conocimientos, con repercusiones negativas en los resultados académicos, también cabe mencionar que los factores laborales y personales juegan un papel protagónico, por tal razón debe ser prioritario identificar a los estudiantes que tiene predisposición al abandono escolar, para realizar seguimientos y así prevenir o mitigar su desvinculación.

Es importante resaltar, que para evitar la desvinculación educativa, es imprescindible que la familia principal centro de socialización proceda a la motivación y el diálogo, así como la unidad educativa debería tratar a los jóvenes como verdaderos sujetos, dando importancia a sus problemas y posibles soluciones.

El docente cumple un papel preponderante en el constructo de la afectividad, según Pérez y Reinoza es frecuente encontrar como el vacío afectivo es substituido por el educador, quienes establecen vínculos afectivos con sus alumnos, constituyéndose en auténticos padres y madres sustitutas ante el hecho que los propios padres no pueden dedicar el tiempo de calidad necesario a sus hijos $(\mathrm{p}, 632)$.

La incertidumbre continúa porque luego los adolescentes, pasan a ser jóvenes, otro proceso de cambio hacia la vida universitaria, transición de la educación media a la superior, donde se preparan para la etapa laboral, es decir tendrán mayores responsabilidades con mayor riesgo a la desmotivación. Es sustancial hacer conciencia que estudiar es un 
trabajo, que requiere esfuerzo y sacrificio, tanto de los progenitores como de sus hijos, aunque en ocasiones la situación económica repercute en el estudiante, porque al no cubrir sus necesidades primarias, buscan la solución en el trabajo remunerado, porque la familia hace exigencias económicas para el hogar, ayuda en labores doméstica, el desempleo, estudiantes que tienen hijos, y la disminución de ofertas laborales agravan su problema.

En conclusión las causas del fracaso o éxito escolar, es amplio porque va desde lo personal a lo sociocultural y en la mayoría la mezcla de factores personales con sociales.

\section{DISCUSIÓN}

El objetivo de esta revisión bibliográfica pretende determinar las tendencias sociales relevantes que conllevan al fenómeno de la desidia estudiantil. Se considera que la educación, es la formación prioritaria y básica de los individuos, aunque muchas personas atribuyen que la misma está determinada por la unidad educativa en la cual se ha educado. Pero en realidad no es así, porque la primera escuela de formación es el hogar, conformada por la familia.

La Familia.

La familia es responsable de la enseñanza de los hijos y está integrada por un grupo de familiares unidos por vínculos afectivos en las cuales existen normas y disciplinas que cumplir, donde se aprenden valores, cultura e identidad. Se considera que es funcional, cuando sus miembros viven armónicamente, dialogan, solucionan problemas, influyendo mucho en la personalidad del adolescente. Sin embargo, algunos progenitores no asumen el papel de cuidadores o guías de sus hijos, más bien, delegan estas funciones a miembros familiares o amistades. En cambio otros endilgan toda la responsabilidad a la escuela, cuando la obligación debe ser de los padres, pero ellos justifican de diferentes formas, aduciendo que su obligatoriedad en el trabajo es importante para la economía de su hogar y en otros casos simplemente afirman no tener tiempo.

Cabe mencionar, que existen diferentes criterios conceptuales de la familia considerada como: la responsable de la formación social primaria, para su posterior socialización externa y no tan solo social; También como el sitio de aprendizaje emocional, económico, valores y que actualmente está impactado por la inserción laboral de los progenitores, para poder suplir las necesidades económicas para bienestar de la familias, por tal razón no van a poder cumplir con los objetivos de formación. Todo estos cambios en la familia, permiten asumir responsabilidades no tan solo sociales, sino económicas indispensables para el cuidado y la continuidad de sus estudios, caso contrario habrán dificultades que conmocionaría el papel fundamental que cumple, en la formación y guía de sus hijos. Los conflictos familiares son efectos negativos al núcleo de la familia, tendrá consecuencias de tipo socio- familiar, que repercutirá en los hijos ocasionando falta de afecto familiar y desorientación emocional. La falta de comunicación, origina deterioro de ese núcleo, originando trastornos 
del comportamiento, porque sin diálogo, ni afectos, el resultado será una familia desligada, en que cada miembro actúa de forma independiente, sin contar el apoyo de unos u otros miembros. .

.Por tal razón, es necesario aclarar el rol fundamental que debe cumplir la familia , cuya obligación es, satisfacer las necesidades Psico-socio económicas de sus miembros, mientras que la escuela, se encarga de la formación de aptitudes, o capacidad intelectual que tiene el alumno para alcanzar sus logros, de ello dependerán las actitudes, o sea la perseverancia, motivación, sacrificio, esfuerzo e interés y la metodología, serían las estrategias herramientas e instrumentos que facilitan el aprendizaje.

Los Jóvenes.

Durante el desarrollo de la vida, niños, adolescentes y jóvenes, sufren el proceso evolutivo de cambios en la búsqueda de su identidad, que justamente está basado en los problemas familiares, sociales, económicos y escolares, que pueden generar un desequilibrio Psico-emocional, ocasionando cambios de conducta.

El período de la adolescencia, es la etapa de transición entre la niñez y la adultez, durante este período ocurren grandes cambios físicos, fisiológicos conductuales emocionales y sociales, que pueden llegar a la autonomía, siendo el momento preciso en que requieren la mayor atención y comprensión de sus progenitores. Las emociones son indefinibles, se consideran que tienen diferentes orígenes, además son involuntarias, que pueden causar, cambios de humor, como la respuesta a un estímulo ocasionando un efecto psicosomático. Se conoce que las emociones pueden ser: Positivas, consideradas todo lo que es agradable o satisfactorio; y las negativas, todo aquello que nos resulte desagradable o insatisfactoria, a la vez, pueden ser temporales, de corta o larga duración. En tanto que el afecto, referido a los sentimientos, tienen menor duración. Peter Salovey y John Mayer en 1990.

Entre las emociones más comunes tenemos el estrés, la desmotivación y la frustración, por ello, urge la necesidad, que los jóvenes sean tratados y controlados, para evitar que se conviertan en agresivos, introvertidos, indisciplinados, depresivos o con trastornos de ansiedad, con claras manifestaciones, de sentirse solos y acorralados, ya sea por recibir constantes regaños en el hogar y centro de estudios donde se los acusa diariamente de todo lo malo que ocurra, haciéndolos sentirse incomprendidos. La situación es preocupante, por lo que requieren ayuda Profesional, que alguien los guie, los escuche y en caso de no ser atendidos, sentirán una fuerte carga emocional que a través de su rostro se refleja la angustia, manifestando la ayuda que piden. Sin embargo, existen personas que agravan el estado Psico-emocional del adolescente, al sobredimensionar la situación por la cual atraviesan, ocasionando inseguridad, desgano y la consecuente desinterés por el estudio, bajo rendimiento académico y la deserción escolar que cada día se incrementa.

En la actualidad, un gran porcentaje de estudiantes no se interesen por la educación impartida durante el proceso enseñanza- aprendizaje , parece ser que el único propósito que tienen es hacer presencia, culminar sus estudios pero de una manera fácil, sin el mayor esfuerzo posible , 
considerando como un simple compromiso, porque en realidad no disfrutan del placer en adquirir nuevos conocimientos. $Y$ es que gran parte de la humanidad, se han convertido en personas conformistas, sin valores éticos ni morales, en que solo les interesa obtener el dinero de una u otra forma, con la finalidad de cumplir determinados caprichos, sin importar como obtengan esos recursos económicos, dejando de lado las buenas enseñanzas, prejuicios, moral, etc. Sin embargo, una minoría, con objetivos claros, adopta la vida con responsabilidad, manteniendo una mirada fija de seguridad hacia un futuro digno y próspero que lo conducirá al éxito y la satisfacción por el cumplimiento de sus metas.

Las autoridades educacionales, están muy preocupadas por las consecuencias que conllevan a la deserción de los educandos y en su afán de brindar solución al problema, insisten en hacer cambios permanentes en los sistemas educativos. En la actualidad, se renuevan carreras técnicas, mallas curriculares, actividades, escuelas para padres, atención psicológica, pero lo cierto es que no hay respuesta positiva, persistiendo el total desinterés por los estudios, tratando de justificar que tienen problemas en la asimilación del aprendizaje y en determinados casos se responsabiliza al docente, por falta de experiencia pedagógica, incomprensión, entre otros factores.

Dentro de toda actividad educativa, el docente cumple un rol importante, porque mediante sus conocimientos, trata de llegar al estudiante, para que desarrollen habilidades cognitivas y emocionales, creando habilidades que les permita ser independiente, y poder actuar en un momento determinado de forma general y satisfactoria. Es importante reconocer que la mayor parte de los docentes tienen objetivos claros que son: educar, formar, motivar y socializar experiencias, ya que el proceso de enseñanza aprendizaje tiene herramientas apropiadas para contrarrestar esta desmotivación, útiles para extraer de ese letargo al estudiante, para inducirlo a la búsqueda de lo cognitivo y la investigación con la finalidad de superar el desgano estudiantil.

Es frecuente encontrar, maestros con el perfil de orientación extrínseca hacia el alumno, caracterizado porque dentro sus enseñanzas, no proporcionan objetivos de la asignatura dictada, no crean valores ni actitudes, ni hacen retroalimentación, selecciona a los alumnos, simplemente se interesa por cumplir con el programa de estudio a como dé lugar, sin importar los vacíos dejados en los conocimientos, por esa razón, tienen muchos alumnos reprobados y justifican su actitud censurando a los estudiantes, sin asumir sus propios errores, es decir, sólo preocupa su calificación como educador. Sin embargo, Valdez, Román, Cubillas y Moreno en un estudio de investigación realizado en la Universidad de Costa Rica, manifiestan que entre las causas de la deserción, está la situación familiar, económica, falta de pedagogía del docente, asignaturas inútiles, falta de diálogo- comprensión y sanciones drásticas por el menor motivo (p. 122).

Síndrome de Burnout

En forma general, se comenta mucho del aburrimiento o desánimo que suelen, presentar, trabajadores, docentes, estudiantes y muchas personas 
por realizar actividades importantes en la vida. Pues bien, se trata del síndrome de Burnout, conocido, como cansancio, agotamiento físico y mental, que ha sido motivo de interés por muchos investigadores. Tal es el caso de algunos educandos que han adquirido conocimientos durante el proceso de formación, pero de pronto podrían sentir desgaste de energía y agotamiento, en cambio, otros que no han podido desarrollar el intelecto ni cumplir sus objetivos, adoptan la decisión de abandonar sus ideales, sin enfrentar la realidad agravando sus problemas, ocasionándoles insatisfacción.

El Estrés.

El término Estrés surge de los años 30 s, un joven llamado Hans Selye, estudiante de medicina observó que los enfermos que atendía presentaban síntomas comunes y generales como: decaimiento, falta de apetito, baja de peso, ansiedad, esto llamó su atención y le denominó Síndrome de estar enfermo . Otros autores consideran que el estrés es la alteración o estado de alerta para enfrentar una situación excepcional.

En términos generales, el estrés, es un problema frecuente en todas las personas pero en este caso, al referimos a los adolescentes, ellos suelen ser muy afectados por los problemas propios del hogar y la escuela, que adicionado a su inmadurez e indecisión, adoptarán actitudes de incomprensión que lo conducirán a la desmotivación por la educación y superación. De igual forma, la educación superior, afecta emocionalmente de forma negativa a los jóvenes, debido a la frecuente presión recibida de manera prolongada, ya sean por exageradas tareas, retro alimentación, carga horaria, días de clases, adicionado a los problemas personales y laborales, que pueden ocasionar el síndrome de Burnout, con la consecuente afectación de su salud, físico-psíquico-emocional.

Redes sociales.

Resulta complicado conocer que a nivel mundial, las redes sociales, se han convertido en un medio de comunicación sencillo oportuno y práctico, utilizado por personas de todas las edades y en la educación, ha sido considerada como principal herramienta de trabajo en la investigación, comunicación con sus docentes, familiares y amistades, pero así también es uno de los motivos por la cual distrae mucho la atención de los alumnos, complicando la problemática, porque el alumno pierde el interés por atender las clases, el aprendizaje , obligaciones y disposiciones a cumplir, pero efecto el más grave, es la falta de comunicación personal con la familia, ya sea en el hogar, en una reunión, paseo, o distracción, que trae como resultado, las familias desligadas. Aparte de esto se indica, que las redes cuidan la identidad de la información pero 2013 señala que hay leyes de las redes sociales que garantizan la privacidad de datos de identificación, sin embargo, existen personas que aceptan como amistades a personas extrañas para que ingresen a su perfil, en ese momento se pierde la privacidad, y está sujeto a muchos riesgos personales y familiares. El exagerado uso del internet, puede resultar adictivo y afecta a las relaciones interpersonales, ya que solamente se comunican es a través de las redes. En definitiva, el 
estudio realizado nos señala que el móvil y el internet, ocasionan ansiedad, depresión, autoestima baja y trastornos conductuales.

Malos hábitos sociales

El estudio identificó, que los malos hábitos que adoptan ciertos jóvenes, están relacionados, con la malas compañías, o aquellos que proceden de familias disfuncionales, pues ambas situaciones van a generar graves cuadros de morbilidad, violencia, drogas, pandillas, delincuencia, es decir conductas de riesgo, con los consecuentes problemas legales, familiares y escolares que los conducen al abandono escolar.

\section{Referencias}

Rueda, G. (08 de febrero de 2014). Ecuador consume 16 millones de kilos de uva anualmente. El Telegrafo. Recuperado de https://www.eltelegrafo.com.ec/noticias/economia/8/ ecuador-consume-16-millones-de-kilos-de-uva-anualmente

Wright, G. (2018). Agronegocios. Ecuador: Agronegocios. Recuperado de http://agronegociosecuador.ning.com/page/conoce-la-primera-vina-de

Johnson, H., \& Robinson , J. (2017). ATLAS MUNDIAL DEL VINO (7ma ed.). España: Blume

Palacios, A. (2016). MITOS Y LEYENDAS DEL VINO (Primera ed.). España: AMV Ediciones

GUTIERREZ, L. (2017). LOS NUEVOS VIÑADORES. España: Planeta.

PAADIN, L., \& PAADIN, A. (2017). LAS PIEDRAS QUE HACIAN VINO. España: SERVINO.NET.

Agronegocios. (1 de marzo de 2018). Conoce la primera viña de Ecuador reconocida internacionalmente. México: Agronegocios. Recuperado de http://agronegociosecuador.ning.com/page/conoce-la-primera-vina-de

Winery, C. E. (10 de marzo de 2018). Nosotros. Ecuador: MetaMorf Net Media. Recuperado de http://www.chaupiestancia.com/espanol/nosotros.html.

Telégrafo, E. (8 de febrero de 2014). Ecuador consume 16 millones de kilos de uva anualmente. El telégrafo. Recuperado de https://www.eltelegrafo.com.ec/noticias/economia/8/ ecuador-consume-16-millones-de-kilos-de-uva-anualmente.

Magliona Markovicth, C. \& Lo\#pez Medel, L. (1999). Delincuencia y Fraude Informático. Santiago de Chile: Jurídica de Chile.

Camacho Losa, L. (1987). El Delito Informático. Madrid: L. Camacho.

Acevedo Esparza. (2010). Tecnología e Informática. Ecuador: Colegio Técnico Industrial José Elias Puyana.

Cuervo Alvarez, J. (2014). Informática Jurídica. Recuperado de http://www.informatica-juridica.com/trabajos/delitos-informaticosproteccion-penal-de-la-intimidad/

Téllez Valdés,J. (2008). Derecho informático. México: McGraw-Hill.

Miró Llinares,F. (2012). El cibercrimen. Madrid: Marcus Felson.

Alain Ambrosi, Valérie Peugeot \& Daniel Pimienta. (2005). Enfoques Multiculturales sobre las Sociedades de la Información. Francia: C\&F.

Levene, R. \& Levene, R. (2005). DerechoEcuador. Recuperado de https:// www.derechoecuador.com/delitos-informaticos 
Daniel Behar Rivero. (2008). Metodología de la Investigación. Argentina: Shalom.

Hernández Sampieri,R., Fernández Collado, C. \& Baptista Lucio, M.P. (2010). METODOLOGÍA de la investigación. México: McGraw-Hill.

Fiscalia del Gobierno Ecuatoriano. (2015). Fiscalia General del Estado Ecuador. Recuperado de https://www.fiscalia.gob.ec/los-delitos-informaticos-vandesde-el-fraude-hasta-el-espionaje/

Alcívar Trejo,C., Domenech Alvarez, G. \& Ortìz Chimbo,K. (2015). LA SEGURIDAD JURÍDICA FRENTE A LOS DELITOS INFORMÁTICOS. Revista de Investigación Jurídica, 10(12), 48 - 55.

Ministerio de Justicia y Derechos Humanos Presidencia de la Nación. (de 2008). InfoLEG. Recuperado de http://servicios.infoleg.gob.ar/infolegInternet/ anexos/140000-144999/141790/norma.htm

MPF. (2014). Ministerio Publico Fiscal de la Ciudad Autonoma de Buenos Aires. Recuperado de http://delitosinformaticos.fiscalias.gob.ar/wp-content/ uploads/2014/02/CyberCrime-Informe-

Alcaldía Mayor de Bogotá. (2009). Régimen Legal De Bogotá D.C. Recuperado de http://www.alcaldiabogota.gov.co/sisjur/normas/ Norma1.jsp?i=34492

Asamblea Nacional. (2014). LA ASAMBLEA NACIONAL DE LA REPÚBLICA BOLIVARIANA DE VENEZUELA. Recuperado de http://web.archive.org/web/20140902120028/http:// www.tsj.gov.ve/legislacion/ledi.htm 